\title{
COMPLEX APPROXIMATION OF REAL FUNCTIONS BY RECIPROCALS OF POLYNOMIALS
}

\author{
DANIEL WULBERT
}

\begin{abstract}
Characterizations are given for local and global best rational approximations to a real function. The characterizations are specialized to reciprocals of polynomials, where they are used to settle some conjectures and questions.
\end{abstract}

\section{INTRODUCTION}

Let $f$ be a continuous real valued function defined on a real interval. Let $r$ be a real valued rational function in $R_{n}^{m}$. If $r$ is a local best approximation to $f$ from $R_{n}^{m}$, it is a global best approximation. If the same $r$ is also a local best approximation to $f$ from the complex rational functions $R_{n}^{m}(\mathbf{C})$, it is not known if $r$ must be a global best approximation from $R_{n}^{m}(\mathbf{C})$. To state known results let

$$
\begin{gathered}
\operatorname{crit}(f-r)=\{x:|(f-r)(x)|=\|f-r\|\}, \\
R_{\text {crit }}=\left\{\frac{p}{q}: p \in P_{m}, q \in P_{n}, \text { and }\{x: q(x)=0\} \cap \operatorname{crit}(f-r)=\varnothing\right\}, \quad \text { and } \\
f_{c}=c f+(1-c) r, \quad c \in \mathbf{R} .
\end{gathered}
$$

An oscillation of length $k$ is an increasing subset $\left\{x_{j}\right\}_{j=1}^{k}$ of $\operatorname{crit}(f-r)$ for which $(f-r)\left(x_{j}\right)=-(f-r)\left(x_{j+1}\right)$. With these definitions we outline the framework relevant to this paper: From the classical theory, $r=\frac{p}{q}$ is a best approximation to $f$ from $\mathbf{R}_{n}^{m}$ if and only if there is an oscillation of length $\max \{m+\operatorname{deg} q+n+\operatorname{deg} p\}+2$ [Cheney, 1966]. If there is an oscillation of length $m+n+2$, then for all sufficiently small $c>0, r$ is a best approximation to $f_{c}$ from $R_{n}^{m}(\mathbf{C})$ [Wulbert, 1980]. If there is an oscillation of length $m+2 n+2$, then $r$ is a best approximation to $f$ from $\Re R_{n}^{m}(\mathbf{C})$, and consequently is the unique best approximation to $f_{c}$ for all $c$ [Wulbert, 1978]. A local best approximation to $f_{c}$, for all positive $c$, is a best approximation to $f$ from $\mathfrak{R} R_{n}^{m}(\mathbf{C})$ [Wulbert, 1988]. A local best approximation to $f$ from $R_{n}^{m}(\mathbf{C})$, is a best approximation on $\operatorname{crit}(f-r)$ to $f$ from $R_{\text {crit }}$. This fact was known to A. Ruttan [Ruttan, 1985]. We will also include a short proof below [Lemma 4].

Suppose $\operatorname{card}(\operatorname{crit}(f-r))=m+n+2$. If $r$ is a best approximation on $\operatorname{crit}(f-r)$ to $f$ from $R_{\text {crit }}$ then $r$ is a global best approximation to $f$ from

Received by the editors May 11, 1988 .

1980 Mathematics Subject Classification (1985 Revision). Primary 41A27. 
$R_{n}^{m}(\mathbf{C})$. In particular in this setting if $r$ is a local best approximation, then $r$ is a global best approximation [Ruttan, 1985].

With the potential application of his result to the local-global approximation problem above, Ruttan stated the following conjectures: for a real valued rational function $r$

[i] $r$ is a best approximation (local or global) to $f$ from $R_{n}^{m}(\mathbf{C})$ if and only if $r$ is a best approximation to $f$ from $R_{\text {crit }}$, on $\operatorname{crit}(f-r)$.

[ii] If $r$ is a best approximation to $f$ from $R_{n}^{m}(\mathbf{C})$ then $f-r$ has an oscillation of length $m+n+2$ on which $r$ is the best approximation to $f$.

The results also suggest the following questions:

[iii] If $f-r$ has an oscillation of length $m+n+2$ but not one of length $m+2 n+2$, then there is a $c^{*}>0$ such that $r$ is a best approximation to $f_{c}$ for $c<c^{*}$ but it is not a best approximation for $c^{*}<c$. Is there a characterization of such a $c^{*}$ ?

[iv] If $r$ is a best approximation from $R_{\text {crit }}$ to $f_{c}$ for all $c>0$ is $r$ a best approximation to $f$ from $R_{n}^{m}(\mathbf{C})$ ?

[v] Is Ruttan's result true if the condition on the cardinality of the critical point set is reduced to assuming that the maximal length of an oscillation is $m+n+2$ ?

This work presents a characterization of best (and local best) approximations. The characterization is specialized to the case $m=0$ (reciprocals of polynomials) to show that conjectures [i] and [ii] are false, that the answer to questions [iv] and [v] are no, and to give bounds to the $c^{*}$ of [iii].

\section{NOTATION AND DEFINITIONS}

The real and complex numbers are represented with $\mathbf{R}$ and $\mathbf{C}$ respectively. All functions are defined on subsets of the real numbers and are normed with the supremum norm. The polynomials (resp., with complex coefficients) of degree less than or equal to $n$ are written $P_{n}$ (resp., $\left.P_{n}(\mathbf{C})\right)$. The polynomials that are positive on an interval domain are written $P_{n}^{+}$. Also put

$$
R_{n}^{m}=\left\{\frac{p}{q}: p \in P_{m}, q \in P_{n}^{+}\right\} .
$$

We assume that if $\frac{p}{q} \in R_{n}^{m}$, then $p$ and $q$ have no common factors. We define $R_{n}^{m}(\mathbf{C})$ analogously. For a function $f$

$$
\operatorname{crit}(f)=\{x:|f(x)|=\|f\|\} \quad \text { and } Z(f)=\{x: f(x)=0\}
$$

are called the critical point set and the zero set of $f$. For a subset of functions $S$,

$$
\operatorname{dist}(f, S)=\inf \{\|f-s\|: s \in S\} .
$$

A member $s$ in $S$ is a best approximation to $f$ if

$$
\|f-s\|=\operatorname{dist}(f, S) .
$$


If there is a neighborhood $U$ of $s$ such that $s$ is a (unique, resp.) best approximation to $f$ from $U \cap S$, then $s$ is a (strict, resp.) local best approximation to $f$ from $S$. If $r$ is a strict local best approximation to $f_{c}$ for $0 \leq c<1$ then $r$ is a waning local best radiating term for $f$. The expression will often be abbreviated to waning local best approximation or by using first letters. The real and imaginary parts of a function $f$ are abbreviated $\mathfrak{R} f$ and $\mathfrak{I} f$, and

$$
\mathfrak{R} S=\{\mathfrak{R} s: s \in S\} .
$$

The term an oscillation of length $k$ and the symbol $R_{\text {crit }}$ are defined in the introduction.

\section{GeNERAL CHARACTERIZATIONS}

Let $\frac{p}{q}$ be the best approximation to a continuous real function $f$ from $R_{n}^{m}$. In this section we give characterizations for $\frac{p}{q}$ being a local best approximation from $R_{n}^{m}(\mathbf{C})$, a global best approximation from $R_{n}^{m}(\mathbf{C})$, or a best approximation from $R_{\text {crit }}$. The results will be specialized later, and used throughout the paper.

Proposition 1 (global characterization). At a critical point of $f-\frac{p}{q}$, the following are equivalent:

$$
\begin{gathered}
\left|f-\frac{u+i s}{v+i t}\right| \leq\left\|f-\frac{p}{q}\right\|, \\
\frac{u+i s}{u+i t}=\frac{p}{q} \quad \text { or } \quad 2\left(f-\frac{p}{q}\right) \mathfrak{R}\left[\frac{p(u+i s)}{(q u-p v)+i(q s-p t)}-\frac{p}{q}\right] \geq \frac{p^{2}}{q^{2}},
\end{gathered}
$$

and

$$
2\left(f-\frac{p}{q}\right) p[s(q s-p t)+u(q u-p v)] \geq\left[(q s-p t)^{2}+(q u-p v)^{2}\right] \frac{p}{q}\left[2 f-\frac{p}{q}\right] .
$$

The equivalence of the corresponding statements is true if the inequalities are replaced everywhere with either strict inequalities or with equalities.

Proof. Proceeding from line (1.1) we compute

$$
\begin{aligned}
\left\|f-\frac{p}{q}\right\| & \geq\left|f-\frac{u+i s}{v+i t}\right| \\
= & \left\{\left[\left(f-\frac{p}{q}\right)-\left(\frac{p}{q}-\mathfrak{R} \frac{u+i s}{v+i t}\right)\right]^{2}+\left[\mathfrak{T} \frac{u+i s}{v+i t}\right]^{2}\right\}^{1 / 2} \\
= & \left\{\left\|f-\frac{p}{q}\right\|^{2}+2\left(f-\frac{p}{q}\right)\left[\frac{u v+s t}{v^{2}+t^{2}}-\frac{p}{q}\right]\right. \\
& \left.+\left[\frac{u v+s t}{v^{2}+t^{2}}-\frac{p}{q}\right]^{2}+\left[\frac{s v-t u}{v^{2}+t^{2}}\right]^{2}\right\}^{1 / 2} .
\end{aligned}
$$


Squaring both sides and manipulating the equation a little we get

$$
\begin{aligned}
2\left(f-\frac{p}{q}\right)\left[(u v+s t) q-p\left(v^{2}+t^{2}\right)\right] & \\
& \geq \frac{\left[(u v+s t) q-p\left(v^{2}+t^{2}\right)\right]^{2}+[s v-u t]^{2} q^{2}}{\left[v^{2}+t^{2}\right] q}, \\
& =\frac{\left[(p t-q s)^{2}+(p v-q u)^{2}\right]\left[v^{2}+t^{2}\right]}{\left[v^{2}+t^{2}\right] q} .
\end{aligned}
$$

Now

$$
\frac{u+i s}{v+i t}=\frac{p}{q} \quad \text { if and only if }\left[(p t-q s)^{2}+(p v-q u)^{2}\right]=0 .
$$

Hence assuming this term is not equal to 0 , we divide by it to obtain

$$
\begin{gathered}
2\left(f-\frac{p}{q}\right) \frac{p^{2} q u v+p^{2} q s t-p^{3} v^{2}-p^{3} t^{2}}{\left[(p t-q s)^{2}+(p v-q u)^{2}\right] q} \geq \frac{p^{2}}{q^{2}}, \\
2\left(f-\frac{p}{q}\right) \frac{p q[s(q s-p t)+u(q u-p v)]-p\left[(p t-q s)^{2}+(p v-q u)^{2}\right]}{\left[(p t-q s)^{2}+(p v-q u)^{2}\right] q} \geq \frac{p^{2}}{q^{2}}, \\
2\left(f-\frac{p}{q}\right)\left[\frac{p s[q s-p t]+p u[q u-p v]}{[p t-s q]^{2}+[q u-p v]^{2}}-\frac{p}{q}\right] \geq \frac{p^{2}}{q^{2}} .
\end{gathered}
$$

This proves that (1.1) implies (1.2), and the argument is reversible. To prove that (1.1) is equivalent to (1.3) we proceed from (1.1) to equation (1.4) and obtain

$$
\begin{gathered}
2\left(f-\frac{p}{q}\right)\left\{p[s(q s-p t)+u(q u-p v)]-\frac{p}{q}\left[(p t-q s)^{2}+(p v-q u)^{2}\right]\right\} \\
\geq \frac{p^{2}}{q^{2}}\left[(p t-q s)^{2}+(p v-q u)^{2}\right], \\
2\left(f-\frac{p}{q}\right) p[s(q s-p t)+u(q u-p v)] \geq\left[(p t-q s)^{2}+(p v-q u)^{2}\right] \frac{p}{q}\left[2 f-\frac{p}{q}\right] .
\end{gathered}
$$

Again the argument is reversible, also the only inequality in the proof occurs in the initial use of equation (1.1).

We remind the reader that the definition of waning local best radiating term for $f$ (abbreviated to wlbrt or to waning local best approximations) is given in the notation section.

Lemma 2 (local characterization). $\frac{p}{q}$ is not a waning local best approximation to $f$ if and only if there exist $u, s \in P_{m}$ and $v, t \in P_{n}$ for which

$$
2\left(f-\frac{p}{q}\right) p[s(q s-p t)+p(q u-p v)]>(p t-q s)^{2} \frac{p}{q}\left(2 f-\frac{p}{q}\right)
$$

on $\operatorname{crit}\left(f-\frac{p}{q}\right)$. 
Proof. Suppose that the inequality holds. Then for points in $\operatorname{crit}\left(f-\frac{p}{q}\right)$ and for sufficiently small $\lambda$ :

$$
\begin{aligned}
2\left(f-\frac{p}{q}\right) p[ & \left.s(q s-p t)+\left(p-\lambda^{2} u\right)(p v-q u)\right] \\
> & {\left[(p t-q s)^{2}+\lambda^{2}(q u-p v)^{2}\right] \frac{p}{q}\left(2 f-\frac{p}{q}\right) . }
\end{aligned}
$$

An application of the general characterization of Proposition 1 shows that

$$
\frac{p-\lambda^{2} u+i \lambda s}{q-\lambda^{2} v+i \lambda t}
$$

is a better approximation to $f$ than $\frac{p}{q}$.

To prove the necessity of the inequality, we use Lemma 7 and Lemma 9 with the word "strict" corrected to "waning" from [Wulbert, Illinois J. Math., 1980]. If $\frac{p}{q}$ is not a local best approximation to $f$ there must be $\gamma \in P_{n}, \delta \in P_{m}$, and $k \in P_{m+n}$ such that

$$
2\left(f-\frac{p}{q}\right) \frac{k q-p \gamma^{2}}{q^{3}}>\frac{(p \gamma-q \delta)^{2}}{q^{4}},
$$

or

$$
\begin{gathered}
2\left(f-\frac{p}{q}\right) p\left[k p q-p^{2} \gamma^{2}\right]>\frac{(p \gamma-q \delta)^{2} p^{2}}{q}, \\
2\left(f-\frac{p}{q}\right) p\left[q^{2} \delta^{2}+k p q-2 p q \gamma \delta-(p \gamma-q \delta)^{2}\right]>\frac{(p \gamma-q \delta)^{2} p^{2}}{q} .
\end{gathered}
$$

Since $p$ and $q$ are relatively prime we can choose the polynomials $s, t, u$, and $v$ so that $s=\delta, t=\gamma$, and $q u-p v=k-s t$. Continuing the inequalities,

$$
\begin{aligned}
& 2\left(f-\frac{p}{q}\right) p\left[q^{2} s^{2}-p q s t-p^{2} q v+p q^{2} u-(p t-q s)^{2}\right]>\frac{(p t-q s)^{2} p^{2}}{q}, \\
& 2\left(f-\frac{p}{q}\right) p\left[s(q s-p t)+p(q u-p v)-\frac{(p t-q s)^{2}}{q}\right]>(p t-q s)^{2} \frac{p^{2}}{q^{2}}
\end{aligned}
$$

and finally

$$
2\left(f-\frac{p}{q}\right) p[s(q s-p t)+p(q u-p v)]>(p t-q s)^{2} \frac{p}{q}\left(2 f-\frac{p}{q}\right) .
$$

Proposition 3. Let $\frac{a}{b} \in R_{\text {crit }}$, then on $\operatorname{crit}\left(f-\frac{p}{q}\right)$

$$
\left\|f-\frac{p}{q}\right\|>\left|f-\frac{a}{b}\right|
$$

if and only if

$$
2\left(f-\frac{p}{q}\right) p a(q a-p b)>(q a-p b)^{2} \frac{p}{q}\left[2 f-\frac{p}{q}\right] .
$$


The corresponding statements with the inequality " $\geq$ " are also equivalent. Proof. This is a corollary to Proposition 1 obtained by putting $u=a, v=b$, $s=0$, and $t=0$.

Lemma 4. Let $\frac{a}{b} \in R_{\text {crit }}$, then on $\operatorname{crit}\left(f-\frac{p}{q}\right)$

$$
\left\|f-\frac{p}{q}\right\|>\left|f-\frac{a}{b}\right|
$$

implies

$$
\left\|f-\frac{p}{q}\right\|>\left|f-\frac{p+i \lambda a}{q+i \lambda b}\right| .
$$

The corresponding implication with the inequality " $\geq$ " is also true.

Proof. This follows from Proposition 1 by putting $u=p, v=q, s=\lambda a$, $t=\lambda b$, and comparing the result with the statement of Proposition 3 above. However a direct proof is to observe that on $\operatorname{crit}\left(f-\frac{p}{q}\right)$ the range of the linear fractional transformation

$$
\lambda \rightarrow \frac{p+i \lambda a}{q+i \lambda b}
$$

is a circle symmetric about the real axis. The end points-and hence the entire circle- of the diameter that lies on the real axis are both within a distance $\left\|f-\frac{p}{q}\right\|$ of $f$.

The following is from [Wulbert, Illinois J. Math., 1980]:

Lemma 5. $\frac{p}{q}$ is a local best approximation to $f$ if and only if it is a local best approximation on $\operatorname{crit}\left(f-\frac{p}{q}\right)$.

Proposition 6. If $\frac{a}{b} \in R_{\text {crit }}$, and on $\operatorname{crit}\left(f-\frac{p}{q}\right)$

$$
\left\|f-\frac{p}{q}\right\|>\left|f-\frac{a}{b}\right|
$$

then $\frac{p}{q}$ is not a local best approximation to $f$ from $R_{n}^{m}(\mathbf{C})$. Proof. This is immediate from Lemmas 4 and 5.

\section{CHARACTERIZATION FOR APPROXIMATION BY RECIPROCALS OF POLYNOMIALS}

When a characterizations of the last section are specialized to approximations from $R_{n}^{0}$, the equations simplify, and the comparisons between the types of approximation become more compelling.

Proposition 7. On $\operatorname{crit}\left(f-\frac{1}{q}\right), \frac{1}{q}$ is respectively not:

(7.1) a best approximation to $f$ from $R_{n}^{0}(\mathbf{C})$,

(7.2) a unique best approximation to $f$ from $R_{n}^{0}(\mathbf{C})$,

(7.3) a waning local best approximation to $f$ from $R_{n}^{0}(\mathbf{C})$, 
(7.4) a best approximation to $f$ from $R_{\text {crit }}$,

(7.5) a unique best approximation to $f$ from $R_{\text {crit }}$; if and only if there are nonzero $v$ and $t$ in $P_{n}$ such that

$$
2\left(f-\frac{1}{q}\right)\left[\frac{v}{v^{2}+t^{2}}-\frac{1}{q}\right]>\frac{1}{q^{2}}
$$

$$
2\left(f-\frac{1}{q}\right)\left[\frac{f}{v^{2}+t^{2}}-\frac{1}{q}\right] \geq \frac{1}{q^{2}}, \text { or } \quad v^{2}+t^{2}=0 ;
$$

$$
2\left(f-\frac{1}{q}\right)\left[\frac{v}{t^{2}}-\frac{1}{q}\right]>\frac{1}{q^{2}}
$$

$$
2\left(f-\frac{1}{q}\right)\left[\frac{1}{v}-\frac{1}{q}\right]>\frac{1}{q^{2}}, \quad \text { or } \quad v=0
$$

$$
2\left(f-\frac{1}{q}\right)\left[\frac{1}{v}-\frac{1}{q}\right] \geq \frac{1}{q^{2}}
$$

or, equivalently:

$$
\begin{aligned}
& 2\left(f-\frac{1}{q}\right) v>\left[v^{2}+t^{2}\right] \frac{1}{q}\left(2 f-\frac{1}{q}\right) \\
& 2\left(f-\frac{1}{q}\right) v \geq\left[v^{2}+t^{2}\right] \frac{1}{q}\left(2 f-\frac{1}{q}\right)
\end{aligned}
$$

$$
2\left(f-\frac{1}{q}\right) v>t^{2} \frac{1}{q}\left(2 f-\frac{1}{q}\right)
$$

$$
\begin{aligned}
& 2\left(f-\frac{1}{q}\right) v>v^{2} \frac{1}{q}\left(2 f-\frac{1}{q}\right) ; \\
& 2\left(f-\frac{1}{q}\right) v \geq v^{2} \frac{1}{q}\left(2 f-\frac{1}{q}\right) ;
\end{aligned}
$$

Proof. The equivalences of the best approximations from $R_{n}^{0}$, and $R_{\text {crit }}$ follow from Propositions 1 and 3. In Proposition 1 replace $u$ with $1, s$ with 0 , and $q-v$ with $v$. In Proposition 3 replace $q-b$ with $v$. To show the equivalences for local approximation suppose that $\frac{1}{q}$ is not a local best approximation. From Lemma 2 there are $u, s \in \mathbf{R}$ and $\delta, \gamma \in P_{n}$ for which

$$
2\left(f-\frac{1}{q}\right)[s(q s-\delta)+(q u-\gamma)]>(\delta-q s)^{2} \frac{1}{q}\left(2 f-\frac{1}{q}\right) .
$$

Then equation (7.3) is satisfied with

$$
v=s(q s-\delta)+(q u-\gamma) \text { and } t=(q s-\delta) .
$$


Conversely if equation (7.3) is satisfied we select $s$ and $\delta$ so that $\delta-q s=t$. Then choose $u$ and $\gamma$ so that $q u-\gamma=v-s(q s-\delta)$.

\section{TRANSITION APPROXIMATIONS}

If $r$ is a best approximation to a continuous real $f$, from $R_{n}^{m}$, but not from $\mathfrak{R} R_{n}^{m}(\mathbf{C})$ then there is a positive $c^{*}$ such that $c<c^{*}$ implies that $r$ is the unique best approximation to $f_{c}=c f+(1-c) r$, from $R_{n}^{m}(\mathbf{C})$ and for $c>c^{*}$ it is not. There is also such a number for local best approximations.

Illustrations. Let $r(x) \equiv x$ be in $R_{1}^{1}$ and let $f$ be a continuous real function such that

$$
f(x)= \begin{cases}\frac{-3}{2}, & \text { if } x=-2, \\ \frac{-3}{2}, & \text { if } x=-1 \\ \frac{3}{2}, & \text { if } x=1, \\ \frac{3}{2}, & \text { if } x=2 .\end{cases}
$$

Also let $\operatorname{crit}(f-r)=\{-2,-1,1,2\}$. Then the $c^{*}$ discussed above is equal to 1 .

For a second setting in $R_{1}^{0}$ let the rational function be $\frac{3}{x}$, the critical point set be $\{1,3,6\}$, and the continuous function take the values $\left\{2,2,-\frac{1}{2}\right\}$ there. Again the transition number is 1 . In both settings when $c^{*} \geq 1, r$ is not the unique best approximation to $f$ on the critical point set from $R_{\text {crit }}$. In the first setting $\frac{2}{x}$ becomes a best approximation, as does $\frac{3}{4-x}$ in the second.

In this section we give some estimates of $c^{*}$ and some properties of transition approximations (defined below). We start by considering approximations that maximize the left side of the inequalities of the characterization theorems.

Definition. For $a, k \in C(X)$, and $h \in M \subset C(X)$, we will say that $a$ has $h$ as a (local) furthest point from $M$ with respect to $k$ if

$$
\min _{x \in X} k(x)(a-h)(x) \geq \min _{x \in X} k(x)(a-m)(x) \text { for all } m \text { in } M
$$

( $m$ in some $M$ neighborhood of $h$, resp.)

This is not a standard use of the term furthest point. The term has been used for the function that maximizes the maximum absolute values on $X$. The expression here maximizes the minimum values on $X$.

Lemma 8. Let $F$ map a Banach space, $B$, into $C(X)$, and let $y$ and $k$ be in $C(X)$. If $F$ is differentiable at $x$, and $F(x)$ is a local furthest point to $y$ from $F(B)$, with respect to $k$; then 0 is a furthest point to $y-F(x)$ from $F^{\prime}(x)(B)$, with respect to $k$.

Proof. First assume that $k=1$. Now suppose $b$ is in $F^{\prime}(x)(B)$, and

$$
\min [y-F(x)-b]>\min [y-F(x)]=d=\max _{m \in F^{\prime}(B)} \min _{z \in X}[y-m](z) .
$$


Choose an $h$ in $B$ so that $F^{\prime}(x)(h)=b$. From the assumption of differentiability,

$$
\lim _{\lambda \rightarrow 0} \frac{\left\|F(x+\lambda h)-F(x)-\lambda F^{\prime}(x)(h)\right\|}{|\lambda h|}=0 .
$$

Put $\varepsilon=\min [y-F(x)-b]-d$ and choose $\lambda>0$ small enough that the expression on the left of line $(8.1)$ is less than $\varepsilon /\|h\|$. Now there is some point in $X$ at which $[y-F(x+\lambda h)]<d$. At this point of $X$ we have

$$
\begin{aligned}
\varepsilon & >\frac{\left\|F(x+\lambda h)-F(x)-\lambda F^{\prime}(x)(h)\right\|}{\lambda} \\
& \geq \frac{\|[y-F(x)-\lambda b]-[y-F(x-\lambda h)]\|}{\lambda} \\
& \geq \frac{\min [y-F(x)-\lambda b]-d}{|\lambda|} \\
& \geq \frac{(1-\lambda) \min [y-F(x)]+\lambda \min [y-F(x)-b]-d}{\lambda} \\
& \geq \frac{(1-\lambda) d+\lambda \min [y-F(x)-b]-d}{\lambda} \\
& \geq \min [y-F(x)-b]-d=\varepsilon .
\end{aligned}
$$

Replacing $y$ and $F$ with $k y$ and $k F$, shows that the case for the weighted furthest point follows from the proven case with weight $=1$.

Comment. We defined furthest point without using absolute values. So multiplying everything by -1 we obtain a corresponding theorem using max instead of $\mathrm{min}$ in the definition of furthest point. Had we used absolute values in the definition we could prove the result by replacing the square brackets in the above argument with absolute values. However the statement for using maximums of absolute values is different. The corresponding statement says that 0 is a best approximation to $y-F(x)$ from $F(x)(B)$.

For $u, v, s$, and $t$ in $P_{n}$ put

$$
H(u, v, s, t)=\operatorname{Re} \frac{p[u+i s]}{[q u-p v]+i[s q-p t]}-\frac{p}{q} .
$$

\section{Lemma 9.}

$$
\begin{aligned}
& H^{\prime}(u, v, s, t)[y, z, w, x] \\
& \quad=p \frac{(t w-s x-v y+u z)\left([q u-p v]^{2}-[s q-p t]^{2}\right)+(-v w+u x-t y+s z)(q u-p v)(s q-p t)}{\left\{\left([q u-p v]^{2}-[s q-p t]^{2}\right)^{2}+([q u-p v][s q-p t])^{2}\right\}^{2}} .
\end{aligned}
$$

Proof. The verification consists of computing the partial derivatives, using the partials to compose the derivative, taking the real part, and collecting terms in an appropriate way. We only will list the partials. 


$$
\begin{aligned}
& \frac{\partial H}{\partial s}(w)=\frac{p t-i p v}{[(p u-p v)+i(s q-p t)]^{2}} w \\
& \frac{\partial H}{\partial t}(x)=\frac{-p s+i p u}{[(p u-p v)+i(s q-p t)]^{2}} x, \\
& \frac{\partial H}{\partial y}(y)=\frac{-p v-i p t}{[(p u-p v)+i(s q-p t)]^{2}} y, \\
& \frac{\partial H}{\partial z}(z)=\frac{p u+i p s}{[(p u-p v)+i(s q-p t)]^{2}} z .
\end{aligned}
$$

Lemma 10. If $t \geq 0$ and $v /\left(v^{2}+t^{2}\right)$ is a furthest point from $1 / q(x)$ from $\mathfrak{R} R_{n}^{0}$ with respect to $2(f(x)-1 / q(x)) q^{2}(x)$, then $t \equiv 0$.

Proof. From Lemma 8, $k v /\left(v^{2}+t^{2}\right)$ is a furthest point from $k \frac{1}{q}$ implies that $k\left[v /\left(v^{2}+t^{2}\right)-1 / q\right]$ has 0 as a furthest point from $k H^{\prime}[v, t](z, y)$, where $H(v, t)=v /\left(v^{2}+t^{2}\right)$. The derivative of $H$ is equal to $\left(t^{2}-v^{2}\right) z-2 v t y$ divided by a positive term. Now choose $\lambda \in P_{n}$ so that,

$$
\operatorname{sgn} \lambda=v^{2}-q v, \quad \text { on } Z(t) \cap \operatorname{crit}\left(f-\frac{1}{q}\right),
$$

and

$$
|\lambda|\left|t^{2}-v^{2}\right|<|t|\left|\left(t^{2}+v^{2}\right)-v q\right| \text { off } Z(t)
$$

The right side of (ii) is greater than zero unless $v /\left(v^{2}+t^{2}\right)=1 / q$. We will show that this cannot happen. Since $v /\left(v^{2}+t^{2}\right)$ is a furthest point it follows from the definition of furthest point and Proposition 7 that if at a critical point $v /\left(v^{2}+t^{2}\right)=1 / q$, then $1 / q$ is the best approximation to $f$. In fact it must be the best approximation to $\lambda f+(1-\lambda) \frac{1}{q}$ for all $\lambda>0$. This however implies that $\frac{1}{q}$ is the unique best approximation to $f$ from $\mathfrak{R} R_{n}^{0}$ [Wulbert, 1988]. In this case the furthest point is obtained only when $v \equiv q$ and $t \equiv 0$. For if any other function were a furthest point, on $\operatorname{crit}(f-r)$, it would be zero or have the same sign as $f(x)-\frac{1}{q(x)}$. Thus contradicting the unique best approximation property of $\frac{1}{q(x)}$. But in (ii) we assumed a critical point not in $Z(t)$ so $t \not \equiv 0$. Hence the right side of (ii) is greater than zero, and we can find a $\lambda$ satisfying (i) and (ii) above. In the above derivative of $H$ put $z=t-\lambda$, and $y=\frac{q}{2}-v$. The numerator of the derivative becomes

$$
t\left[\left(t^{2}+v^{2}\right)-v q\right]-\lambda\left[t^{2}-v^{2}\right],
$$

and has the same sign as $\left\{v /\left(v^{2}+t^{2}\right)-1 / q\right\}$. It follows that $k\left[v /\left(v^{2}+t^{2}\right)-1 / q\right]$ does not have 0 as a furthest point from $k H^{\prime}[v, t](z, y)$.

Definition. Let

$$
c^{*}=\max \left\{c \in \mathbf{R}: \frac{p}{q} \text { is a best approximation to } f_{c}=c f+(1-c) \frac{p}{q}\right\} .
$$


A best approximation $\left(\neq \frac{p}{q}\right)$ to $f_{c^{*}}$ will be called a transition approximation for $f$, and $c^{*}$ is the transition number or transition constant associated with $f$.

Proposition 11. If $r$ is a wlbrt for $f$ and $c^{*}<1$ then $f$ has a transition approximation. Also, if $\frac{p}{q}$ is a local but not global best approximation to $f$, then $f$ has a transition approximation.

Proof. For all $c>c^{*}$ let $r_{c}$ be a best approximation to $f_{c}$. Since as $c$ approaches $c^{*}, r_{c}$ is a minimizing sequence for $f_{c^{*}}$. It has a subsequence that converges almost everywhere to a best approximation to $f_{c^{*}}$. Since $\frac{p}{q}$ is a strict local best approximation to $f_{c}$ for all $c^{*} \leq c<1$, this best approximation is not $\frac{p}{q}$.

Put

$$
\Gamma=\Re \frac{p(\alpha+i \beta)}{(q \alpha-p \delta)+i(q \beta-p \gamma)} ; \quad \alpha, \beta \in P_{m} \text { and } \delta, \gamma \in P_{n} .
$$

Lemma 12. If $(u+i s) /(v+i t) \neq p / q$ is a transition approximation to $f$ on $X=\operatorname{crit}\left(f-\frac{p}{q}\right) \backslash Z(p)$, then $\mathfrak{R} p(u+i s) /((q u-p v)+i(s q-p t))$ is a furthest point to $\underset{q}{p}$ from $\Gamma$ with respect to $2(f-p / q) q^{2} / p^{2}$.

Proof. Suppose that

$$
r=\frac{\alpha+i \beta}{\gamma+i \delta} \in R_{n}^{m}, \quad \rho=\Re \frac{p(\alpha+i \beta)}{(q \alpha-p \delta)+i(q \beta-p \gamma)},
$$

but

$$
\begin{aligned}
D & =\min _{x \in X} 2\left(f-\frac{p}{q}\right) \frac{q^{2}}{p^{2}}\left(\rho-\frac{p}{q}\right) \\
& >\min _{x \in X} 2\left(f-\frac{p}{q}\right) \frac{q^{2}}{p^{2}}\left(\Re \frac{p(u+i s)}{(q u-p v)+i(s q-p t)}-\frac{p}{q}\right)=d .
\end{aligned}
$$

Since $(u+i s) /(v+i t)$ is a best approximation to $f$, it follows from the characterization theorem that $d \geq 1$. Choose $1 / D<\lambda<1 / d$, and put $f_{\lambda}=\lambda f+(1-\lambda) p / q$. Using the characterization theorem again we have that, on $X$,

$$
\left|f_{\lambda}-r\right|<\left\|f_{\lambda}-\frac{p}{q}\right\|<\left\|\frac{u+i s}{v+i t}-f_{\lambda}\right\| .
$$

Hence,

$$
\begin{aligned}
\|f-r\| & \leq\left\|f_{\lambda}-r\right\|+\left\|f_{\lambda}-f\right\| \\
& <\left\|f_{\lambda}-\frac{p}{q}\right\|+(1-\lambda)\left\|f-\frac{p}{q}\right\| \\
& \leq \lambda\left\|f-\frac{p}{q}\right\|+(1-\lambda)\left\|f-\frac{p}{q}\right\|,
\end{aligned}
$$

and $r$ is a better approximation to $f$ than is $(u+i s) /(v+i t)$. 
Proposition 13. If $t \geq 0$ and $1 /(v+i t)$ is a transition approximation from $R_{n}^{0}$ to $f$ on $\operatorname{crit}\left(f-\frac{1}{q}\right)$, then $t \equiv 0$.

Proof. The result follows from Lemma 12 and Lemma 10.

\section{ESTIMATES OF TRANSITION NUMBERS}

To estimate the transition numbers we divide the critical point set into its parts with positive error and with negative error. Let

$$
\text { crit }^{+}=\operatorname{crit}\left(f-\frac{1}{q}\right) \cap\left\{x: f(x)>\frac{1}{q(x)}\right\},
$$

and

$$
\text { crit }^{-}=\operatorname{crit}\left(f-\frac{1}{q}\right) \cap\left\{x: f(x)<\frac{1}{q(x)}\right\} \text {. }
$$

Put

$$
f_{c}=c f+(1-c) \frac{1}{q} \text {. }
$$

Proposition 14. [1] $f_{c}$ has $\frac{1}{q}$ as a unique best approximation if

$$
c<\frac{\min _{\text {crit }^{-}} \frac{1}{q}}{2\left\|f-\frac{1}{q}\right\|} .
$$

[2] $f_{c}$ has $\frac{1}{q}$ as a best approximation if

$$
c \leq \frac{\min _{\text {crit }^{-}} \frac{1}{q}}{2\left\|f-\frac{1}{q}\right\|}, \quad \text { and }
$$

[3] $\frac{1}{q}$ is not a local best approximation to $f_{c}$ if

$$
c>\frac{\max _{\text {crit }^{-}} \frac{1}{q}}{2\left\|f-\frac{1}{q}\right\|}, \text { and } \operatorname{card}\left(\mathrm{crit}^{+}\right) \leq n .
$$

Proof. Suppose first that

$$
c<\frac{\min _{\text {crit }^{-}} \frac{1}{q}}{2\left\|f-\frac{1}{q}\right\|},
$$

then for $x \in \mathrm{crit}^{-}$

$$
2 c\left\|f-\frac{1}{q}\right\|<\frac{1}{q}, \quad \frac{2}{q}-2 f_{c}<\frac{1}{q}, \quad \text { and } 0<2 f_{c}-\frac{1}{q} .
$$

Now if there is another function that is as good an approximation to $f_{c}$ as is $\frac{1}{q}$ then there must exist $t$ and $v$ in $P_{n}$ such that

$$
2\left(f_{c}-\frac{1}{q}\right)(q-v) \geq\left(2 f_{c}-\frac{1}{q}\right)\left(t^{2}+(q-v)^{2}\right),
$$

and not both $t$ and $q-v \equiv 0$ (Proposition 7). Since the right side of equation (14.1) is nonnegative, $q-v$ is successively nonpositive and nonnegative at the critical points. This, of course, cannot occur unless $q \equiv v$. For if $w \in P_{n}$ is 
chosen so that $\operatorname{sgn} w=\operatorname{sgn}\left(f-\frac{1}{q}\right)$ at the critical points where also $q=v$, then for small $\lambda, q-v+\lambda w$ would be a nonzero polynomial which both has degree $n$, and has $n$ sign changes. But if $q \equiv v$, then since $0<2 f_{c}-\frac{1}{q}$, we also have from equation (14.1) that $t \equiv v$.

Proving [2] is similar to the above proof. In this setting $2 f_{c}-\frac{1}{q}$ is nonnegative instead of positive. However the existence of a better approximation implies that equation (14.1) can be satisfied with a strict inequality replacing the weak inequality there (Proposition 7). Now the same contradiction occurs as in the proof of [1].

To prove the last assertion we wish to find $v$ and $t$ in $P_{n}$ so that

$$
2\left(f_{c}-\frac{1}{q}\right)(q-v) \geq\left(2 f_{c}-\frac{1}{q}\right)\left(t^{2}\right),
$$

on $\operatorname{crit}\left(f-\frac{1}{q}\right)$ (Proposition 7). Similar to the above argument, the conditions of this assertion imply that $2\left(f_{c}-\frac{1}{q}\right)$ is negative on crit ${ }^{-}$. So we can satisfy the above inequality if we replace $t$ with $\lambda t$ for sufficiently large $\lambda$.

Proposition 15. Let $k$ be a constant function. If $\operatorname{card}[\operatorname{crit}(f-k)] \leq 2 n+1$ then $k$ is a local best approximation to $f$ if and only if $k$ is a global best approximation.

Proof. Suppose that $k$ is not the best approximation to $f$. From Proposition 7, there are $v$ and $t$ in $P_{n}$ such that

$$
2(f-k)\left(\frac{v}{v^{2}+t^{2}}-k\right) \geq k^{2} \text {. }
$$

We will show that the set crit $^{+}$of Proposition 14 has card $\left(\mathrm{crit}^{+}\right) \leq n$. Now $v-k\left(v^{2}+t^{2}\right)$ changes sign with $f-k$. But $v-k\left(v^{2}+t^{2}\right)$ has at most $2 n+1$ sign changes, and is negative for all real numbers of sufficiently large absolute value. Therefore it can have at most $n$ points in the set crit $^{+}$. Since obviously for a constant $q, \max _{\text {crit }^{-}} \frac{1}{q}=\min _{\text {crit }^{-}} \frac{1}{q}$ the results follows from Proposition $14,[2]$ and [3].

Proposition 16. If

(i) $q$ has a real zero (e.g., if the degree of $q$ is odd), and

(ii) $f-\frac{1}{q}$ admits a maximal oscillation of length $2 n+1$ for which $n+1$ of the critical points are in the set $\mathrm{crit}^{+}$,

then for all $c>0, f_{c}$ has $\frac{1}{q}$ as a unique best approximation.

Proof. $f_{c}$ has $\frac{1}{q}$ as a unique best approximation for all $c>0$ if and only if $f$ has $\frac{1}{q}$ as a best approximation from the real parts of $R_{n}^{0}$ [Wulbert, 1988]. Hence suppose that $v /\left(v^{2}+t^{2}\right)$ is a better approximation to $f$ than is $\frac{1}{q}$. Then

$$
\operatorname{sgn}\left[\frac{v}{v^{2}+t^{2}}-\frac{1}{q}\right]=\operatorname{sgn}\left[f-\frac{1}{q}\right] \text {. }
$$


We then have that $q v-v^{2}-t^{2}$ oscillates sign on the critical points and has positive sign at the first and last of these. Now $q v-v^{2}-t^{2}$ has $2 n$ zeros dictated by the sign changes on the critical points. The zero of $q$ off the domain of approximation forces one more zero. This shows that $q v=v^{2}+t^{2}$. This is a contradiction.

Example 17. The conclusion of part [1] of Proposition 14 is not true if, in the hypothesis, the inequality " $<$ " is replaced with $\leq$.

To see this let

$$
q=\frac{1}{4}\left(x^{2}+1\right), \quad \operatorname{crit}\left(f-\frac{1}{q}\right)=\{-1,0,1,2\},
$$

and at these critical points respectively put $f=1,5,1, \frac{9}{5}$. Define $v=q$, and $t=x(x-2)$. Either by applying Proposition 7 , or by direct computation, we conclude that $1 /\left(v^{2}+t^{2}\right)$ is as good an approximation to $f$ as $\frac{1}{q}$.

Example 18. The conclusion in part [3] of Proposition 14 is not true if the inequality " $>$ " is replaced with $\geq$ in the hypothesis.

Letting $q$ and $f$ be as defined in the last example, the weak inequality conditions are satisfied with $c=1$. However part [2] of the proposition gives that $\frac{1}{q}$ is a best approximation to $f$.

Example 19. Proposition 16 is not true if $q$ has no real zeros.

The construction of Example 20 will verify this statement.

\section{LOCAL APPROXIMATIONS AND APPROXIMATION FROM $R_{\text {crit }}$}

In this section we present counterexamples to the conjectures and some of the questions listed in the introduction.

Example 20. There is a continuous function $f$ and a polynomial $q$ of degree 2 such that $\frac{1}{q}$ is not a local best approximation to $f$ from $R_{2}^{0}(C)$, but $f_{\lambda}=$ $\lambda f+(1-\lambda) \frac{1}{q}$ has $\frac{1}{q}$ as a best approximation from $R_{\text {crit }}$ on $\operatorname{crit}\left(f-\frac{1}{q}\right)$ for all $\lambda>0$.

Construction. Put

$$
\frac{1}{q}=\frac{1}{3 x^{2}+5.75}
$$

We will construct $f$ so that the critical point set of the error function is $x_{1}=0$, $x_{2}=.1, x_{3}=1.9, x_{4}=2.1, x_{1}=5$. We will need to show that there is no polynomial $\beta$ of degree 2 such that

$$
\operatorname{sgn}\left(\frac{1}{\beta}-\frac{1}{q}\right)\left(x_{j}\right)=(-1)^{j}, \quad j=0,1,2,3,4 .
$$

The verification has three cases. First no $\beta$ that is positive on the critical point set can satisfy (1) above. In that case $q-\beta$ would have to switch sign 4 times, which, of course, cannot be done by a second degree polynomial. Since $\beta$ 
must be positive at $x_{0}, x_{2}$, and $x_{4}$ the other two cases are for $\beta$ obtaining a nonpositive minimum between $x_{0}$ and $x_{2}$, and for $\beta$ obtaining a nonpositive minimum between $x_{2}$ and $x_{4}$. The latter case is easily dismissed. Since such a $\beta$ would necessarily: (1) be decreasing on $\left(-\infty, x_{2}\right),(2)$ be less than $q$ at $x_{0}$, and (3) be greater that $q$ at $x_{1}$. For the remaining case we look at the derivative of $q-\beta$. Looking at its sign at $x_{2}, x_{3}$, and $x_{4}$; the derivative of $q-\beta$ has a zero between $x_{2}$, and $x_{4}$. Also since this derivative is a linear function it is negative to the left of this one zero (a minimum for $q-\beta$ ), and in particular at $x_{0}$. However the derivative of $q$ is zero at $x_{0}$. Also since $\beta$ has a nonpositive minimum between $x_{0}$ and $x_{2}$ it has a negative derivative at $x_{0}$. Since these facts are not compatible, this third case is also unobtainable, and no $\beta$ satisfies the equation above.

Now we construct $f$. For $j=0,1,2,3,4$. Define

$$
f\left(x_{j}\right)=\frac{1}{q\left(x_{j}\right)}+1000(-1)^{j}\left(\frac{1}{q\left(x_{j}\right)}\right)^{2} .
$$

From Proposition 7, $f_{\lambda}$ has $\frac{1}{q}$ as a best approximation from $R_{\text {crit }}$. We will present second degree polynomials $v$ and $t$ such that

$$
2\left(f-\frac{1}{q}\right)\left[\frac{v}{t^{2}}-\frac{1}{q}\right] \geq \frac{1}{q^{2}} \text {. }
$$

Then Proposition 7 shows that $\frac{1}{q}$ is not a local best approximation to $f$ from $R_{2}^{0}(C)$. From our choice of $f$ we need only find $v$ and $t$ such that

$$
(-1)^{j}\left[\frac{v}{t^{2}}-\frac{1}{q}\right] \geq \frac{1}{1000}
$$

Put $v=(x-3)(x-2)$, and $t=(x-2)\left(x+\frac{1}{2}\right)$. So we simply verify that $\left[v / t^{2}-1 / q\right]$ has the following values at $x_{0}$ through $x_{4}$ respectively:

$$
.2500,-.6643,1.8188,-1.4060, .0087,
$$

and this completes the construction.

For the example below, given a function $f$ put $f_{c}=c f+(1-c) 1$.

Example 21. There is a function $f$ with the following properties:

[1] for all sufficiently small $c>0, f_{c}$ has 1 as a best approximation from $R_{1}^{0}(\mathbf{C})$,

[2] 1 is not a local best approximation to $f$,

[3] 1 is a best approximation to $f$ from $R_{\text {crit }}$,

[4] 1 is not a best approximation to $f_{c}$ from $R_{\text {crit }}$ for all $c$,

[5] 1 is a local best approximation to $f_{c}$ if and only if it is a global best approximation. 
Construction. Let $\varepsilon>0$, and put

$$
f(x)= \begin{cases}\frac{1}{2}, & x= \pm 1 \\ \frac{3}{2}, & x= \pm \sqrt{\frac{\varepsilon}{2+\varepsilon}} .\end{cases}
$$

Now put

$$
v \equiv \frac{\varepsilon}{1+\varepsilon} \quad \text { and } \quad t(x)=x
$$

Then

$$
\begin{aligned}
-(1+\varepsilon)\left[\frac{v}{t^{2}}-1\right] & =1, \quad x= \pm 1 \\
(1+\varepsilon)\left[\frac{v}{t^{2}}-1\right] & =1, \quad x= \pm \sqrt{\frac{\varepsilon}{2+\varepsilon}}
\end{aligned}
$$

Hence for $\lambda>\varepsilon$,

$$
\begin{aligned}
-(1+\lambda)\left[\frac{v}{t^{2}}-1\right] & >1, \quad x= \pm 1 \\
(1+\lambda)\left[\frac{v}{t^{2}}-1\right]>1, & x= \pm \sqrt{\frac{\varepsilon}{2+\varepsilon}}
\end{aligned}
$$

Equivalently,

$$
\begin{aligned}
-(1+\lambda) v>-\lambda t^{2}, & x= \pm 1 ; \\
(1+\lambda) v>(2+\lambda) t^{2}, & x= \pm \sqrt{\frac{\varepsilon}{2+\varepsilon}} .
\end{aligned}
$$

From Proposition 7, 1 is not a local best approximation to $f_{1+\lambda}$ for any $\lambda>\varepsilon$. The function $f$ in the statement of the example will in fact be of the form $f_{1+\lambda}$ for some $\lambda>\varepsilon$. The argument above proves statement [1]. Since $f_{1+\lambda}-1$ has a maximal oscillation of length 3, statement [1] is satisfied [Wulbert, Illinois J. Math, 1980]. To establish line [3] we want to show that 1 is not a best approximation to $f_{1+\lambda}$ for $\lambda$ slightly larger than $\varepsilon$. That is, from Proposition 7 we need to show there does not exist a linear $w$ such that

$$
\begin{aligned}
-(1+\lambda) w>-\lambda w^{2}, & & x= \pm 1 ; \\
(1+\lambda) w>(2+\lambda) w^{2}, & & x= \pm \sqrt{\varepsilon /(2+\varepsilon) .}
\end{aligned}
$$

We may assume that $w(1)>0$. For this setting, the optimal $w$ will make the equation exact at 1 , and $w(-\sqrt{\varepsilon /(2+\varepsilon)})=0$. That is, among all the linear functions positive at 1 and satisfying the above equations at both 1 and $-\sqrt{\varepsilon /(2+\varepsilon)}$ such a $w$ will give the lowest possible value at $\sqrt{\varepsilon /(2+\varepsilon)}$. Hence we consider

$$
w(x)=\left[\frac{x+\sqrt{\varepsilon /(2+\varepsilon)}}{1+\sqrt{\varepsilon /(2+\varepsilon)}}\right]\left(\frac{\lambda+1}{\lambda}\right) .
$$


We want to verify that the characterizing equation of Proposition 7 cannot be satisfied at $\sqrt{\varepsilon /(2+\varepsilon)}$. Suppose to the contrary that

$$
\frac{1+\lambda}{2+\lambda} \geq w\left(\sqrt{\frac{\varepsilon}{2+\varepsilon}}\right) \text {. }
$$

Equivalently,

$$
\begin{gathered}
\frac{1+\lambda}{2+\lambda} \geq \frac{2 \sqrt{\frac{\varepsilon}{2+\varepsilon}}}{1+\sqrt{\frac{\varepsilon}{2+\varepsilon}}} \frac{\lambda+1}{\lambda}, \\
\lambda+\lambda \sqrt{\frac{\varepsilon}{2+\varepsilon}} \geq(2+\lambda) 2 \sqrt{\frac{\varepsilon}{2+\varepsilon}}, \\
\frac{\lambda}{4+\lambda} \geq \sqrt{\frac{\varepsilon}{2+\varepsilon}} .
\end{gathered}
$$

If $\lambda$ is sufficiently small (for example: $\varepsilon=1$ and $\lambda=2$ ):

$$
\frac{\lambda}{4+\lambda}<\frac{\varepsilon}{2+\varepsilon}<\sqrt{\frac{\varepsilon}{2+\varepsilon}} .
$$

For such $\varepsilon$ and $\lambda$ the characterizing equations cannot be satisfied, and 1 is the best approximation to $f_{1+\lambda}$ from $R_{\text {crit }}$. However for sufficiently large $\lambda$,

$$
\frac{\lambda}{4+\lambda}>\sqrt{\frac{\varepsilon}{2+\varepsilon}} \text {. }
$$

Hence

$$
\frac{1+\lambda}{2+\lambda}>w\left(\sqrt{\frac{\varepsilon}{2+\varepsilon}}\right)>0,
$$

and so by slightly altering $w$ at 1 and $-\sqrt{\frac{\varepsilon}{2+\varepsilon}}$ so that the characterizing equations are satisfied with a strict inequality " $>$ " we have proven [4] as well as [3]. It remains to establish [5] that if 1 is a local best approximation to $f=f_{\lambda}$ from $R_{1}^{0}(\mathbf{C})$ then it is a global best approximation. If 1 is not a global best approximation then from the characterization in Proposition 7 there are linear functions $v$ and $t$ such that on the critical points,

$$
2(f-1) v>(2 f-1)\left(t^{2}+v^{2}\right) \text {. }
$$

Since not both $v$ and $t$ can be zero,

$$
2(f-1) \frac{v}{t^{2}+v^{2}}>(2 f-1) .
$$

Choose points $x_{1}$ and $x_{2}$ such that

$$
-1<x_{1}<-\sqrt{\frac{\varepsilon}{2+\varepsilon}} \text { and } \sqrt{\frac{\varepsilon}{2+\varepsilon}}<x_{2}<1 .
$$

Now choose a linear $T$ so that

$$
T\left(x_{i}\right)=(-1)^{i} \sqrt{v^{2}\left(x_{i}\right)+t^{2}\left(x_{i}\right)} .
$$


Then $T^{2}$ and $t^{2}+v^{2}$ are quadratic polynomials with two common values. Since $T$ has a zero between $x_{1}$ and $x_{2}, T^{2}<v^{2}+t^{2}$ on $\left(x_{1}, x_{2}\right)$ and $T^{2}>v^{2}+t^{2}$ elsewhere. From Proposition 14, $2 f( \pm \sqrt{\varepsilon /(2+\varepsilon)})<0$, and for all critical points,

$$
2(f-1) v>(2 f-1)\left(t^{2}+v^{2}\right)>(2 f-1) T^{2} .
$$

So from Proposition 7, 1 cannot even be a waning local best approximation.

\section{REFERENCES}

1. D. Braess, Nonlinear approximation theory, Springer-Verlag, Berlin, 1986.

2. E. W. Cheney, Introduction to approximation theory, McGraw-Hill, New York, 1966.

3. M. H. Gutknecht and L. N. Trefetha, Real and complex Chebyshev approximation on the unit disk and interval, Bull. Amer. Math. Soc. 8 (1983), 455-459.

4. __ Real is complex rational Chebyshev approximation on an interval, Trans. Amer. Math. Soc. 280 (1983), 555-561.

5. D. J. Newman, Approximation with rational functions, CBMS Regional Conf. Ser. in Math., Amer. Math. Soc., Providence, R.I., 1979.

6. T. J. Rivlin and H. S. Shapiro, A unified approach to certain problems of approximation and minimization, J. Soc. Indust. Appl. Math. 9 (1961), 670-699.

7. A. Ruttan, $A$ characterization of best complex rational approximants in a fundamental case, Constructive Approximation 1 (1985), 287-296.

8. _ The length of the alternation set as a factor in determining when a best real rational approximation is also a best complex rational approximation, J. Approx. Theory 31 (1981), 230-243.

9. J. L. Walsh, The existence of rational functions of best approximation, Trans. Amer. Math. Soc. 33 (1931), 668-689.

10. D. E. Wulbert, The rational approximation of real functions, Amer. J. Math. 100 (1978), 12811317.

11. The characterization of complex rational approximations, Illinois J. Math. 24 (1980), 140-155.

12. Local best rational approximations to continuous functions and the rays they emanate, J. Approx. Theory 52 (1988), 350-358.

Department of Mathematics, University of California at San Diego, la Jolla, CaliFORNIA 92093 